\title{
Predictive Factors of Neutropenia in HIV-Infected Patients with Malignancy Receiving Chemotherapy or Radiotherapy
}

\author{
Apisada Suthepwanon, Patcharin Uttasri, Lakkana Boonyagars*
}

\begin{abstract}
Objective: To determine the predictive factors of neutropenia in human immunodeficiency virus (HIV)-infected patients with malignancy receiving chemotherapy (CMT) or radiotherapy (RT). Materials and Methods: The author conducted a retrospective study on HIV-infected patients with malignancy receiving CMT or RT at Vajira Hospital, Navamindradhiraj University, Thailand, from January 1, 2013 to December 31, 2017. Baseline demographic characteristics, HIV disease data, and cancer data were collected. Results: A total of 210 courses of CMT, concurrent chemoradiation therapy (CCRT), or RT treatments were administered to $39 \mathrm{HIV}$-infected patients with malignancy. Neutropenia occurred in $51(24.3 \%)$ of the 210 treatment courses in $23(60 \%)$ patients. Multivariable analysis revealed that HIV-infected patients with malignancy who received CMT or CCRT (hazard ratio [HR] 10.83, 95\% confidence interval [CI $]$ 1.36-86.05, $\mathrm{p}=0.024)$ and those who received over five cycles of CMT (HR 5.25, 95\% CI 1.10-26.01, p = 0.037) were independently associated with neutropenia. Conclusion: Receiving CMT or CCRT and receiving more than five cycles of CMT are risk factors for neutropenia in HIV-infected patients with malignancy.
\end{abstract}

Keywords: Malignancy- chemotherapy- radiotherapy-HIV- neutropenia

Asian Pac J Cancer Prev, 23 (2), 445-450

\section{Introduction}

A total of 19.3 million new cases of malignancy and 10 million deaths from malignancy were recorded worldwide in 2020. In Thailand, cancer is the leading cause of death in patients aged less than 70 years (Sung et al., 2021). The treatment modalities of malignancy include surgery, radiotherapy (RT), chemotherapy (CMT), targeted therapy, and immunotherapy. In most cases, the treatment mode selected by doctors and patients, especially in immunocompromised cases, depends on the extent of its side effects. Patients infected with human immunodeficiency virus (HIV) are usually concerned with developing neutropenia when receiving treatments that could affect their bone marrow, such as CMT and RT (Stevens et al., 1990; Wang et al., 2006; Weycker et al., 2014).

Inconclusive results on the incidence of CMT-induced neutropenia in patients infected with HIV have been published. A previous study, for example, reported a nearly two-fold increase in risk of developing neutropenia after receiving neoadjuvant CMT for breast cancer in HIVinfected patients compared with uninfected patients (Ngidi et al., 2017). However, another study reported that patients with breast cancer can receive complete CMT without serious toxicity, regardless of HIV status (Langenhoven et al., 2016). A large study in the United States found that the risk of developing neutropenia in patients receiving cancer CMT for all solid and hematologic malignancies is greatest in the first cycle of treatment (Lyman et al., 2011). Another meta-analysis reported the benefit of granulocyte colony-stimulating factors as a primary prophylaxis for cancer patients receiving myelosuppressive CMT (Wang et al., 2015). The effect of treatment for various malignancies, such as CMT and radiation, on the bone marrow, as well as the predictive factors of neutropenia, of HIV-infected patients remains unknown. Therefore, the author conducted the present study to explore the predictive factors of neutropenia in HIV-infected patients with malignancy receiving CMT or RT.

\section{Materials and Methods}

A retrospective chart review included adults (age $>18$ years) HIV-infected patients who underwent CMT, radiation therapy (RT), or concurrent chemoradiation therapy (CCRT) for any solid or hematologic malignancy over a 5-year period from January 2013 to December 2017 at Vajira Hospital was conducted in this work. We excluded patients with incomplete medical records and patients whose final diagnosis was not solid or hematologic malignancy. The study protocol was approved by the Vajira Institutional Review Board of the Faculty of Medicine, Vajira Hospital, Navamindradhiraj 
University (COA 043/2562).

Data on baseline characteristics, including sex, age, body mass index (BMI), Eastern Cooperative Oncology Group (ECOG) performance status, and comorbidities, were collected. Information on each patient's HIV status, such as CD4 cell count, HIV viral load, opportunistic infection, prophylaxis medication, and antiretroviral therapy, as well as cancer data, including type of cancer, stage of cancer, type of treatment, and CMT regimen, were also collected. Finally, factors potentially influencing neutropenia, such as the white blood cell count, albumin level, and renal and hepatic functions before each CMT, $\mathrm{RT}$, or CCRT cycle, were assessed. The time to neutropenia was defined as the length of time from the start of CMT treatment to the diagnosis of neutropenia. The patients' demographic data were collected on the first date of the CMT, RT, or CCRT session. After each CMT, RT, or CCRT session, the patients will be monitored as inpatient or outpatient depending on the patient's conditions. Then the primary outcome was met when neutropenia has occurred. In case of no neutropenia occurred after the first CMT, RT, or CCRT session, the patients will be follow-up and evaluated whether neutropenia has occurred in the next CMT, CCRT, or RT session.

\section{Statistical analysis}

The data were analyzed using Statistical Package for Social Sciences software, version 22.0 (SPSS Inc., Chicago, IL, USA). Continuous variables were presented as means and standard deviations or medians with interquartile ranges, depending on the normality of the variables. Categorical variables were presented as counts and percentages. Factors associated with neutropenia were analyzed using Kaplan-Meier curves, Cox proportional hazard regression analysis, and Pearson's chi-squared test. The risk factors of neutropenia were obtained by calculating the hazard ratios (HRs) and their $95 \%$ confidence intervals (CIs) of the univariable and multivariable Cox regression analyses. Candidate risk factors with $p$-value $<0.1$ in the univariable analysis were subsequently entered into the multivariable model. Pearson's chi-squared test was used to compare the effect of each CMT regimen for each type of cancer on the risk of neutropenia. A p-value of $<0.05$ was considered to indicate statistical significance.

\section{Results}

From January 1, 2013 to December 31, 2017, total of $71 \mathrm{HIV}$-infected patients with solid and hematologic malignancies were included, 23 patients were excluded because insufficient patient information for further analysis. An additional 9 patients were excluded because they were transferred to another hospital/loss to follow-up $(\mathrm{n}=8)$, and the final diagnosis was not solid or hematologic malignancy $(n=1)$. A final total of 39 HIV-infected patients with malignancy were remained in the study with a total of 210 courses of CMT, CCRT, or radiation treatments. The study flow is shown in Figure 1. The total follow-up time was 6,605 person-days, and the mean follow-up time was 169.36 days/person.
Table 1. Characteristics of 39 HIV-Infected Patients with Malignancy

\begin{tabular}{|c|c|}
\hline Characteristic & Patients $(\mathrm{N}=39)(\%)$ \\
\hline \multicolumn{2}{|l|}{ Age (years) } \\
\hline \multicolumn{2}{|l|}{$($ mean $=50.85 \pm \mathrm{SD}=9.69)$} \\
\hline$\geq 55$ & $12(30.8)$ \\
\hline \multicolumn{2}{|l|}{ Sex - no. $(\%)$} \\
\hline Male & $15(38.5)$ \\
\hline Female & $24(61.5)$ \\
\hline \multicolumn{2}{|l|}{ BMI $\left(\mathrm{kg} / \mathrm{m}^{2}\right)$} \\
\hline \multicolumn{2}{|l|}{$($ mean $=19.72 \pm \mathrm{SD}=4.43)$} \\
\hline$<18.5$ & $10(25.6)$ \\
\hline $18.5-22.9$ & $21(53.8)$ \\
\hline$>23$ & $8(20.5)$ \\
\hline \multicolumn{2}{|l|}{ Performance status score by ECOG* } \\
\hline 0 & $9(23.1)$ \\
\hline 1 & $22(56.4)$ \\
\hline 2 & $7(17.9)$ \\
\hline 3 & $1(2.6)$ \\
\hline 4 & $0(0)$ \\
\hline \multicolumn{2}{|l|}{ Co-morbid disease ${ }^{* *}$} \\
\hline None & $19(48.7)$ \\
\hline Diabetes Mellitus & $5(12.8)$ \\
\hline Hypertension & $8(20.5)$ \\
\hline Dyslipidemia & $7(17.9)$ \\
\hline Chronic hepatitis B virus infection & $4(10.3)$ \\
\hline Others & $8(20.5)$ \\
\hline \multicolumn{2}{|l|}{ CD4 cell counts (cells $\left./ \mathrm{mm}^{3}\right)$} \\
\hline \multicolumn{2}{|l|}{$($ median $=256, \mathrm{IQR}=175-387)$} \\
\hline Unsuppressed HIV viral load & $5(12.8)$ \\
\hline \multicolumn{2}{|c|}{ Total white blood cell counts $\left(\mathrm{x} 10^{3}\right.$ cells $\left./ \mathrm{mm}^{3}\right)$} \\
\hline \\
\hline \multicolumn{2}{|c|}{ Absolute neutrophil counts $\left(\times 10^{3}\right.$ cells $\left./ \mathrm{mm}^{3}\right)$} \\
\hline \multicolumn{2}{|l|}{$($ median $=3.2, \mathrm{IQR}=2.3-4.5)$} \\
\hline Zidovudine-containing regimens & $11(28.2)$ \\
\hline Co-trimoxazole use & $12(30.8)$ \\
\hline \multicolumn{2}{|l|}{ Type of Malignancies } \\
\hline Cervix & $10(25.8)$ \\
\hline Breast & $7(17.9)$ \\
\hline Head and neck & $4(10.3)$ \\
\hline Lung & $2(5.1)$ \\
\hline Diffused large B cell lymphoma & $8(20.5)$ \\
\hline Hodgkin's lymphoma & $2(5.1)$ \\
\hline Other*** & $6(15.4)$ \\
\hline \multicolumn{2}{|l|}{ Stage of cancer } \\
\hline Stage I & $6(15.4)$ \\
\hline Stage II & $18(46.2)$ \\
\hline Stage III & $8(20.5)$ \\
\hline Stage IV & $7(17.9)$ \\
\hline
\end{tabular}

* Performance status score by ECOG scale range from 0 (no disability) to 5 (death); **Some patients had more than one co-morbid disease; ***Other: Bladder cancer, hepatocellular carcinoma, rectal cancer, myelofibrosarcoma, endometrium cancer, ovarian cancer, each $=1$; Abbreviation: BMI, Body mass index; ECOG, eastern cooperative oncology group; IQR, interquartile range; SD, standard deviation 


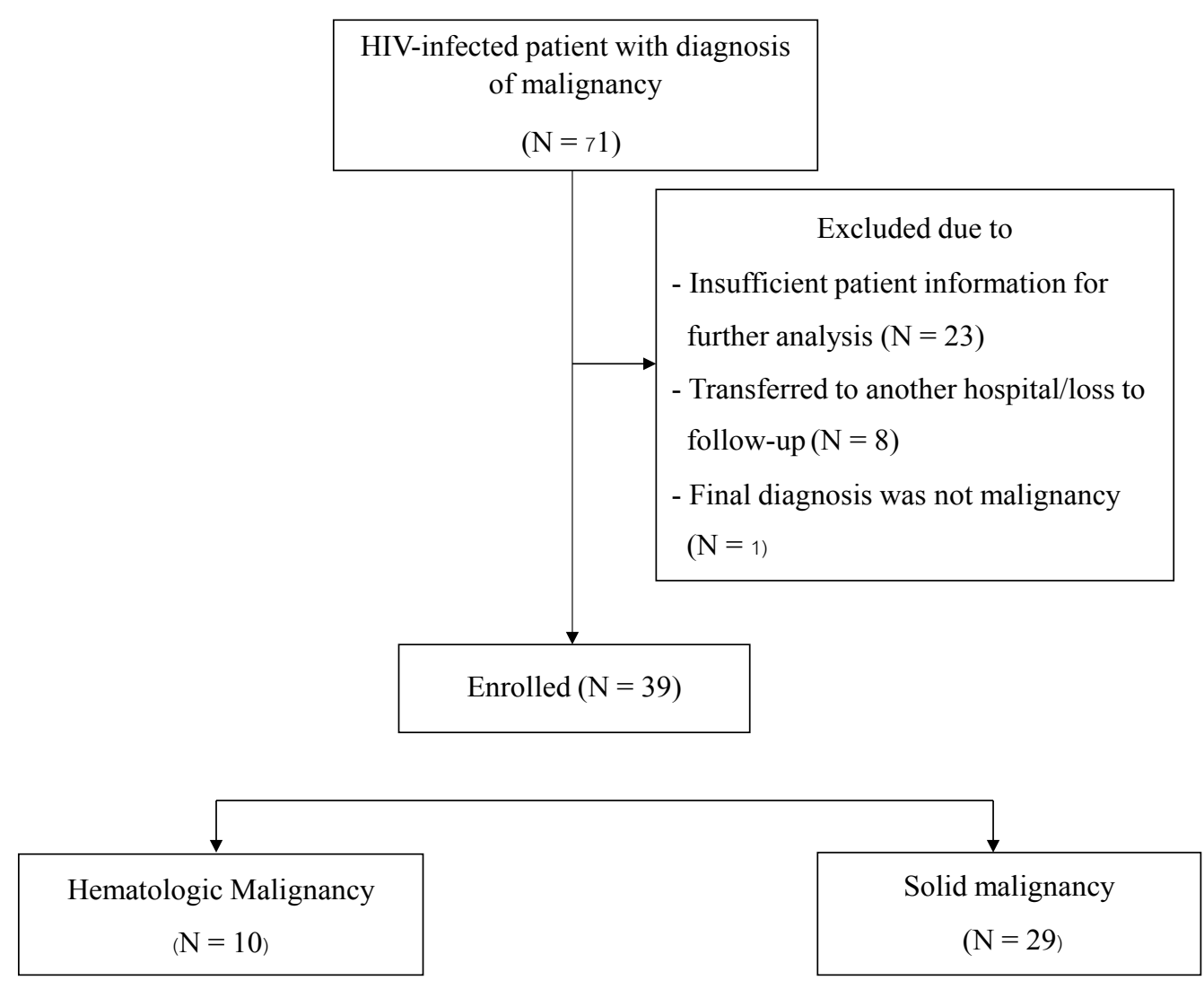

Figure 1. Study Flow

Neutropenia occurred in $51(24.3 \%)$ of 210 treatment courses in $23(59 \%)$ patients. The baseline characteristics of the patients are shown in Table 1. The median age was 50.85 years, and $24(61.5 \%)$ of the patients were female. A total of $31(79 \%)$ patients received ECOG performance status scores less than 1. The median BMI was $19.72 \mathrm{~kg} / \mathrm{m}^{2}$.

The results of the univariable and multivariable analyses of the estimated HRs and 95\% CIs of the patients' characteristics are shown in Table 2 and 3.

Table 2. Univariable Analysis of Risk Factors for Neutropenia

\begin{tabular}{|c|c|c|c|c|}
\hline \multirow[t]{2}{*}{ Characteristic } & \multicolumn{2}{|c|}{ Patients $(\mathrm{N}=39)$} & \multirow[t]{2}{*}{ HR } & \multirow[t]{2}{*}{$95 \% \mathrm{CI}$} \\
\hline & $\begin{array}{c}\text { Neutropenia } \\
n=23(\%)\end{array}$ & $\begin{array}{c}\text { No neutropenia } \\
n=16(\%)\end{array}$ & & \\
\hline Age, mean (SD) & $53.17( \pm 9.42)$ & $46.75( \pm 8.84)$ & - & - \\
\hline$\geq 55$ years & $10(43.5)$ & $2(12.5)$ & 2.72 & $1.11-6.65^{*}$ \\
\hline BMI, mean (SD) & $20.03( \pm 4.23)$ & $21.56( \pm 2.58)$ & - & - \\
\hline Low BMI $\left(\geq 18.5 \mathrm{~kg} / \mathrm{m}^{2}\right)$ & $8(34.8)$ & $2(12.5)$ & 1.65 & $0.69-3.96$ \\
\hline Sex (male) & $11(47.8)$ & $4(25)$ & 1.51 & $0.66-3.44$ \\
\hline Performance status by ECOG & $5(21.7)$ & $3(18.8)$ & 1.21 & $0.45-3.31$ \\
\hline \multicolumn{5}{|l|}{ ECOG 2-3 (vs. 0-1) } \\
\hline Co-morbid disease: Yes (vs. no) & $14(60.9)$ & $6(37.5)$ & 1.73 & $0.74-4.03$ \\
\hline Unsuppressed HIV viral load (vs, suppressed) & $2(8.7)$ & $5(31.3)$ & 0.3 & $0.07-1.25$ \\
\hline CD4 level, median (IQR) & $265(180-478)$ & $250(129-379.3)$ & - & - \\
\hline $\mathrm{CD} 4<100$ cells $/ \mathrm{mm}^{3}$ & $2(8.7)$ & $0(0)$ & 2.62 & $0.33-20.83$ \\
\hline Zidovudine-containing regimens & $6(26.1)$ & $5(31.3)$ & 1.25 & $0.48-3.26$ \\
\hline Co-trimoxazole use & $7(30.4)$ & $5(33.3)$ & 0.88 & $0.36-2.13$ \\
\hline Hematologic malignancies (vs. solid malignancies) & $9(39.1)$ & $1(6.3)$ & 2.22 & $0.96-5.16^{*}$ \\
\hline Stage of cancer: III or IV (vs. I or II) & $13(56.5)$ & $3(18.8)$ & 3.05 & $1.28-7.28^{*}$ \\
\hline CMT/ CCRT (vs. RT alone) & $23(100)$ & $4(25)$ & 46.57 & $1.44-1507.2 *$ \\
\hline Number of cycles of CMT: $\leq 5$ (vs. $>5$ courses) & $6(26.1)$ & $13(81.2)$ & 7.83 & $1.83-33.44 *$ \\
\hline
\end{tabular}

* p-value $<0.1$; Abbreviation: BMI, Body mass index; CCRT, concurrent chemoradiation therapy; CI, confidence interval; CMT, chemotherapy; ECOG, eastern cooperative oncology group; HR, hazard ratio; RT, radiation therapy 


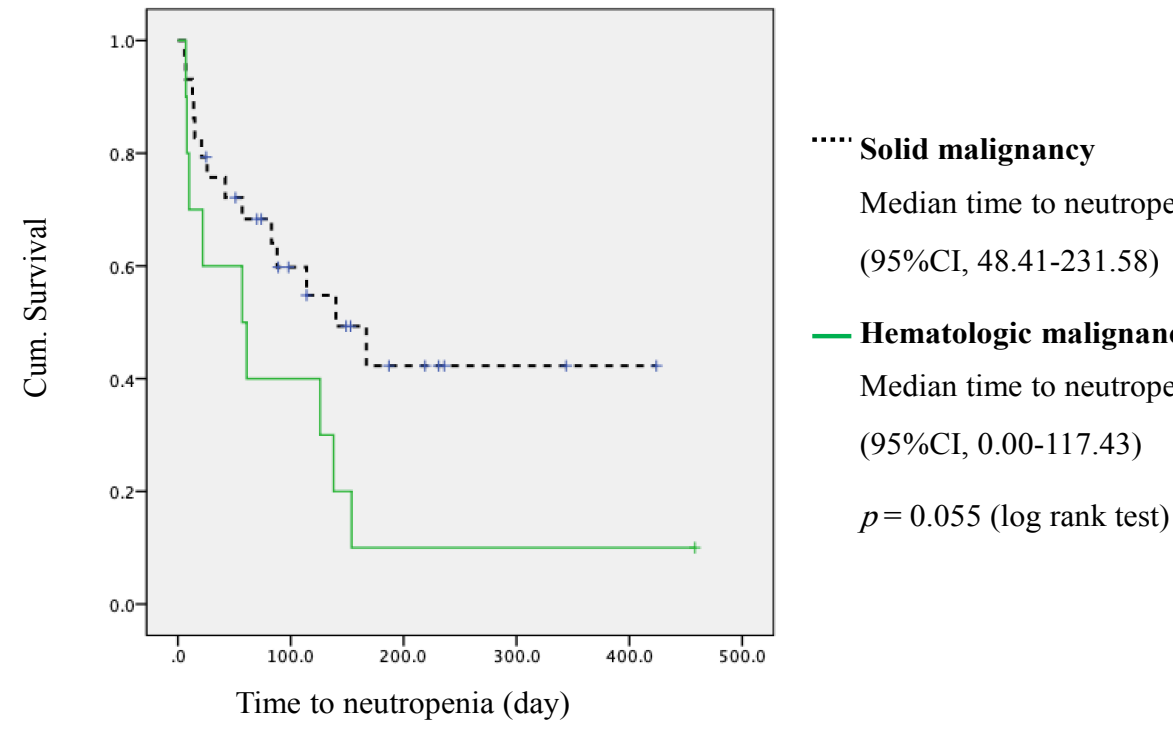

Figure 2. Kaplan-Meier Curve of Time to Neutropenia among Patients with Solid Malignancy and Patients with Hematologic Malignancy.

Table 3. Multivariable Analysis of Risk Factors for Neutropenia

\begin{tabular}{|c|c|c|c|c|}
\hline \multirow[t]{2}{*}{ Characteristic } & \multicolumn{2}{|c|}{ Patients $(\mathrm{N}=39)$} & \multirow[t]{2}{*}{ Adjusted HR } & \multirow[t]{2}{*}{$95 \% \mathrm{CI}$} \\
\hline & $\begin{array}{c}\text { Neutropenia } \\
n=23(\%)\end{array}$ & $\begin{array}{c}\text { No neutropenia } \\
n=16(\%)\end{array}$ & & \\
\hline Age, $\geq 55$ years & $10(43.5)$ & $2(12.5)$ & 1.7 & $0.57-5.07$ \\
\hline Hematologic malignancies (vs. solid malignancies) & $9(39.1)$ & $1(6.3)$ & 1.33 & $0.48-3.71$ \\
\hline Stage of cancer: III or IV (vs. I or II) & $13(56.5)$ & $3(18.8)$ & 1.55 & $0.57-4.21$ \\
\hline CMT/ CCRT (vs. RT alone) & $23(100)$ & $4(25)$ & 10.83 & $1.36-86.05$ \\
\hline Number of cycles of CMT: $\leq 5$ (vs. $>5$ courses) & $6(26.1)$ & $13(81.2)$ & 5.25 & $1.10-26.01$ \\
\hline
\end{tabular}

Abbreviation: CCRT, concurrent chemoradiation therapy; CI, confidence interval; CMT, chemotherapy; HR, hazard ratio; RT, radiation therapy

Univariable analysis revealed that patients with age $\geq 55$ years (HR 2.72, 95\% CI 1.11-6.64, p $=0.029$ ), those with hematologic malignancies (HR 2.22, 95\% CI 0.96-5.16, $\mathrm{p}=0.063$ ), those with stage III or IV cancer (HR 3.05, 95\% CI 1.28-7.28, $\mathrm{p}=0.012$ ), those receiving CMT or CCRT (HR 46.57, 95\% CI 1.44-1507.2, $\mathrm{p}=0.03$ ), and those who received over five cycles of CMT (HR 7.83, 95\% CI 1.83-33.44, $\mathrm{p}=0.005$ ) were likely to develop neutropenia. On the other side, Zidovudine (AZT) and co-trimoxazole use were not associated with neutropenia. The results of the multivariable analysis revealed that receiving CMT or CCRT (HR 10.83, 95\% CI 1.36-86.05, $\mathrm{p}=0.024)$ and receiving over five cycles of CMT (HR $5.25,95 \%$ CI $1.10-26.01, p=0.037$ ) are independent risk factors of neutropenia.

The total number of treatment sessions received by 39 HIV-infected patients with solid and hematologic malignancies was $210(\mathrm{CMT}=128, \mathrm{CCRT}=68$, and $\mathrm{RT}=14$ sessions). The risks of developing neutropenia in different types of cancer and treatment regimens are shown in Table 4. Specifically, the risk of neutropenia was higher in CMT regimens for hematologic malignancies, i.e., diffuse large B-cell lymphoma (odds ratio [OR] 11.49, 95\% CI 1.47-90.90, $\mathrm{p}=0.003$ ) and Hodgkin's lymphoma

Table 4. Analysis of the Risk of Neutropenia Stratified by the Number of Chemotherapy Courses in a Different Type of Cancer

\begin{tabular}{lcccc}
\hline Type of cancer & \multicolumn{2}{c}{ No. of CMT courses (N=190) } & OR & 95\%CI \\
& Neutropenia (49) & No neutropenia (141) & & \\
\hline Regimens used in head and neck cancer (ref) & $1(2)$ & $25(17.7)$ & Ref & - \\
Regimens used in cervical cancer & $2(4.1)$ & $17(12.1)$ & 2.94 & $0.25-34.48$ \\
Regimens used in Lung cancer & $5(10.2)$ & $16(11.3)$ & 7.81 & $0.83-73.16$ \\
Regimens used in diffused large B cell lymphoma & $23(46.9)$ & $50(35.5)$ & 11.49 & $1.47-90.90$ \\
Regimens used in breast cancer & $13(26.5)$ & $26(18.4)$ & 12.5 & $1.52-100$ \\
Regimens used in Hodgkin's lymphoma & $5(10.2)$ & $7(5)$ & 17.86 & $1.78-178.97$ \\
\hline
\end{tabular}

Abbreviation: CI, confidence interval; CMT, chemotherapy; OR, odds ratio; Note: The chemotherapy regimens of some cancer e.g. endometrial cancer $n=1$, ovarian cancer $n=4$, bladder cancer $n=6$, rectal cancer $n=1$, hepatocellular carcinoma $n=2$, sarcoma $n=6$, could not be analyzed in table 4 due to the small numbers. 
(OR 17.86, 95\% CI 1.78-178.97, p = 0.008), compared with that in CMT regimens for head and neck cancer. In addition, CMT regimens for breast cancer (OR 12.5, 95\% CI 1.52-100, $\mathrm{p}=0.005$ ) demonstrated a significantly higher risk of neutropenia compared with CMT regimens for head and neck cancer.

The Kaplan-Meier curves of the time to neutropenia stratified by type of malignancy (hematologic malignancies vs. solid malignancies) are illustrated in Figure 2. The log-rank test revealed marked differences in the time to neutropenia stratified by type of malignancy $(p=0.055)$. The median time to neutropenia in patients receiving CMT regimens for hematologic malignancies was 65 days while that of patients receiving CMT regimens for solid malignancies was 145 days.

\section{Discussion}

The author report the results of a retrospective cohort study involving 39 adult HIV-infected patients with malignancies. Receiving CMT/CCRT and receiving over five cycles of CMT were independently associated with neutropenia. Analyses also showed that patients aged $\geq 55$ years and those with advanced-stage cancer (i.e., stage III or IV) were associated with increased risk of neutropenia, although no statistically significant difference was detected during the multivariable analysis. This finding is consistent with a previous systematic review which found that older age and advanced cancer stage are correlated with higher risks of developing febrile neutropenia among non-HIVinfected patients with cancer receiving CMT (Lyman et al., 2014). Recent studies also reported that receiving CCRT is more highly associated with hematologic toxicity, including neutropenia, compared with receiving RT alone in both HIV-infected and noninfected patients with cancer (He et al., 2018; Vendrell et al., 2018).

Patients who received over five cycles of CMT were significantly associated with neutropenia/febrile neutropenia. A previous study demonstrated that febrile neutropenia is most likely to occur in the first cycle of CMT during four cycles of treatment when patients are treated with full-dose CMT without supportive care (Crawford et al., 2008). However, no report on the number of CMT cycles associated with neutropenia in HIV-infected patients is yet available. To the best of our knowledge, the present study is the first to show that receiving more than five cycles of CMT is a predictive factor of neutropenia.

Our study revealed no association between neutropenia and HIV disease characteristics, such as HIV viral load or CD4 cell count. These results may be explained by the finding that the median total white blood cell and absolute neutrophil counts of the patients prior to receiving CMT in the present study usually exceeded 3,000 cells $/ \mathrm{mm}^{3}$ and 1,500 cells $/ \mathrm{mm}^{3}$, respectively; these values are comparable with the white blood cell and absolute neutrophil counts obtained in studies on immunocompetent patients (Crawford et al., 2008; Lyman et al., 2011; Weycker et al., 2014; Ngidi et al., 2017). In addition, most of the patients in our study demonstrated good control of their HIV status as well as suitable hematologic function prior to cancer treatment.

Interestingly, the CMT regimens used to treat hematologic malignancies demonstrated a greater risk of neutropenia than those regimens used to treat head and neck cancer. Furthermore, the time to neutropenia in hematologic malignancies was shorter than that in solid malignancies. Therefore, patients with hematologic malignancies should be followed-up, and their white blood cell counts should be monitored regularly and frequently. Many previous studies suggested the benefits of prophylactic granulocyte-colony-stimulating factor (G-CSF) treatment for both HIV-infected and noninfected patients with hematologic malignancy receiving CMT or some solid malignancies receiving high-risk regimens, such as dose-dense CMT in breast cancer (Intragumtornchai et al., 2000; Vakkalanka and Link, 2011; Aapro et al., 2017; Ludwig et al., 2019). The author recommends considering prophylactic G-CSF treatment and formulating policies or guidelines for neutropenia treatment and management in HIV-infected patients with malignancy.

The strengths of our study include a wide range of disease severity in our cohort. We also collected information, including laboratory profiles, prior to any treatment initiation. The treatment of HIV infection and malignancy was performed by infectious diseases and oncology specialists. Moreover, because the type of malignancies reported in our study are comparable with the epidemiological data in Thailand, our findings may have some level of generalizability. However, our study also presents a number of limitations. First, incomplete data collection cannot be avoided because of the retrospective nature of the study. In most cases, however, the missing data in our study were not among the main variables of interest. Second, because only a small number of patients in our cohort received radiation, analyses of the associations between radiation site and radiation dose and neutropenia were limited. Prospective data collection may provide us with more accurate data and decrease the effects of these biases.

In conclusion, receiving CMT or CCRT and receiving over five cycles of CMT were predictive risk factors of neutropenia in HIV-infected patients with malignancy. Thus, these patients should be followed-up, and their white blood cell counts should be monitored at frequent and regular intervals.

\section{Author Contribution Statement}

Conceptualization, Boonyagars, L. and Suthepwanon, A.; data collection, Boonyagars, L., Suthepwanon, A. and Uttasri, P.; data validation, Boonyagars, L. and Suthepwanon, A.; data analysis, Boonyagars, L. and Suthepwanon, A.; writing-original draft preparation, Boonyagars, L., Suthepwanon, A. and Uttasri, P.; writingreview and editing, Boonyagars, L. and Suthepwanon, A.; reading and approving the final manuscript, Boonyagars, L., Suthepwanon, A. and Uttasri, P.

Asian Pacific Journal of Cancer Prevention, Vol 23 


\section{Acknowledgements}

The authors would like to thank all of the teams of the Oncology and Infectious Diseases Division in Vajira Hospital, Navamindradhiraj University, Bangkok, Thailand, for their help and support.

\section{Funding Statement}

The present study was supported by the Navamindradhiraj University Research Fund. This study was approved by Vajira Institutional Review Board (COA043/2562).

\section{Conflict of Interest}

The authors declare that there is no conflict of interest in this study. The principal investigator can provide the data of this study if requested.

\section{References}

Aapro M, Bokemeyer C, Ludwig H, et al (2017). Chemotherapyinduced (febrile) neutropenia prophylaxis with biosimilar filgrastim in elderly versus non-elderly cancer patients: Patterns, outcomes, and determinants (MONITOR-GCSF study). J Geriatr Oncol, 8, 86-95.

Crawford J, Dale DC, Kuderer NM, et al (2008). Risk and timing of neutropenic events in adult cancer patients receiving chemotherapy: the results of a prospective nationwide study of oncology practice. J Natl Compr Canc Netw, 6, 109-18.

He Y, Guo T, Guan H, et al (2018). Concurrent chemoradiotherapy versus radiotherapy alone for locoregionally advanced nasopharyngeal carcinoma in the era of intensity-modulated radiotherapy: a meta-analysis. Cancer Manag Res, 10, 1419-28.

Intragumtornchai T, Sutheesophon J, Sutcharitchan P, et al (2000). A predictive model for life-threatening neutropenia and febrile neutropenia after the first course of CHOP chemotherapy in patients with aggressive non-Hodgkin's lymphoma. Leuk Lymphoma, 37, 351-60.

Langenhoven L, Barnardt P, Neugut AI, et al (2016). Phenotype and Treatment of Breast Cancer in HIV-Positive and -Negative Women in Cape Town, South Africa. J Glob Oncol, 2, 284-91.

Ludwig H, Bokemeyer C, Aapro M, et al (2019). Chemotherapyinduced neutropenia/febrile neutropenia prophylaxis with biosimilar filgrastim in solid tumors versus hematological malignancies: MONITOR-GCSF study. Future Oncol, 15, 897-907.

Lyman GH, Abella E, Pettengell R (2014). Risk factors for febrile neutropenia among patients with cancer receiving chemotherapy: A systematic review. Crit Rev Oncol Hematol, 90, 190-9.

Lyman GH, Kuderer NM, Crawford J, et al (2011). Predicting individual risk of neutropenic complications in patients receiving cancer chemotherapy. Cancer, 117, 1917-27.

Ngidi S, Magula N, Sartorius B, et al (2017). Incidence of chemotherapy-induced neutropenia in HIV-infected and uninfected patients with breast cancer receiving neoadjuvant chemotherapy. S Afr Med J, 107, 595-601.

Stevens SK, Moore SG, Kaplan ID (1990). Early and late bonemarrow changes after irradiation: MR evaluation. AJR Am J Roentgenol, 154, 745-50.

Sung H, Ferlay J, Siegel RL, et al (2021). Global Cancer Statistics 2020: GLOBOCAN Estimates of Incidence and Mortality Worldwide for 36 Cancers in 185 Countries. $C A$
Cancer J Clin, 71, 209-49.

Vakkalanka B, Link BK (2011). Neutropenia and neutropenic complications in ABVD chemotherapy for Hodgkin Lymphoma. Adv Hematol, 2011, 656013.

Vendrell I, Ferreira AR, Abrunhosa-Branquinho AN, et al (2018). Chemoradiotherapy completion and neutropenia risk in HIV patients with cervical cancer. Medicine (Baltimore), 97, e11592.

Wang L, Baser O, Kutikova L, et al (2015). The impact of primary prophylaxis with granulocyte colony-stimulating factors on febrile neutropenia during chemotherapy: a systematic review and meta-analysis of randomized controlled trials. Support Care Cancer, 23, 3131-40.

Wang Y, Probin V, Zhou D (2006). Cancer therapy-induced residual bone marrow injury-Mechanisms of induction and implication for therapy. Curr Cancer Ther Rev, 2, 271-9.

Weycker D, Li X, Edelsberg J, et al (2014). Risk of febrile neutropenia in patients receiving emerging chemotherapy regimens. Support Care Cancer, 22, 3275-85.

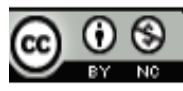

This work is licensed under a Creative Commons AttributionNon Commercial 4.0 International License. 